\title{
Pneumonia redonda, uma apresentação radiológica rara
}

\author{
Round pneumonia, a rare radiological presentation
}

\author{
Rosângela Percinio Gianvecchio ${ }^{1}$, Daniele Munõz ${ }^{2}$, Victor Alexandre P. Gianvecchio ${ }^{2}$, Lilian Albieri ${ }^{3}$
}

\section{RESUMO}

Objetivo: Descrição de caso de pneumonia redonda em criança.

Descrição do caso: Criança do gênero feminino, quatro anos e 11 meses, com dor no corpo, cefaléia e febre sem sinais localizatórios há dois dias. Apresentou radiografia de tórax com imagem redonda em lobo superior do pulmão direito e lobo médio do pulmão esquerdo. O hemograma mostrou leucocitose e neutrofilia. Com a antibioticoterapia, houve resolução do quadro clínico e radiológico.

Comentários: Considerando outros diagnósticos diferenciais diante desta apresentação radiológica, em crianças com quadro infeccioso, é razoável aguardar a resposta clínica à antibioticoterapia antes de prosseguir com investigação de maior complexidade.

Palavras-chave: pneumonia; criança; radiografia torácica.

\section{ABSTRACT}

Objective: To describe a child with round pneumonia.

Case description: A 4-years and 11 months old child presented the following symptoms for about two days: pain in the body, headache and fever. The chest radiography showed a round image at the upper lobe of the right lung and in the medium lobe of the left lung. Leukocytosis with neutrophilia was present in the blood count. Therapy with antibiotics improved the clinical status and the radiological picture.

Comments: Considering the other differential diagnosis and looking at the radiological presentation of a child with the clinical picture of an infectious disease, it is reasonable to test antibiotic therapy prior to perform more invasive diagnostic procedures.

Key-words: pneumonia; child; radiography, thoracic.
${ }^{1}$ Mestre em Pediatria, responsável pelo ambulatório de Infectologia Pediátrico de referência da DIR XIV - Marília e professor do Departamento de Pediatria da Universidade de Marília (Unimar)

${ }^{2}$ Médico pediatra

${ }^{3}$ Residente em Medicina de Família e Comunidade na Faculdade de Medicina da Unimar
Endereço para correspondência:

Rosângela Percinio Gianvecchio

Rua Carlos Botelho, 420

CEP 17515-240 - Marília/SP

E-mail: rosangela@unimedmarilia.com.br

Recebido em: 31/1/2007

Aprovado em: 23/4/2007 


\section{Introdução}

A pneumonia redonda é uma apresentação radiológica atípica de infecção pulmonar, sendo rara em adultos ${ }^{(1)}$. Dos casos de pneumonia, menos de $1 \%$ se manifesta como lesão redonda e, quando ocorre, trata-se de um achado precoce no curso da doença. Por esta razão, muitas vezes, a história de tosse e sintomas respiratórios estão ausentes na apresentação inicial, dificultando o diagnóstico ${ }^{(2)}$.

A tosse, a taquipnéia e a tiragem subcostal são sintomas que indicam o diagnóstico de pneumonia em crianças $^{(3)}$; no entanto, a ausência destes sintomas não afasta o seu diagnóstico. Portanto, a radiografia de tórax se impõe em crianças com toxemia e febre, sem sinais localizatórios. Um estudo a respeito da casuística de pneumonia em um pronto socorro de pediatria, por um período de um ano, encontrou 3.375 casos, representando $15 \%$ dos atendimentos e constituindo a segunda causa de procura do serviço médico de urgência ${ }^{(4)}$.

É, nesse contexto, que se descreve o caso a seguir.

\section{Descrição do caso}

Criança do gênero feminino, com quatro anos e 11 meses de idade, branca, natural e procedente de Marília, foi trazida para atendimento médico com queixa de dor no corpo, febre e

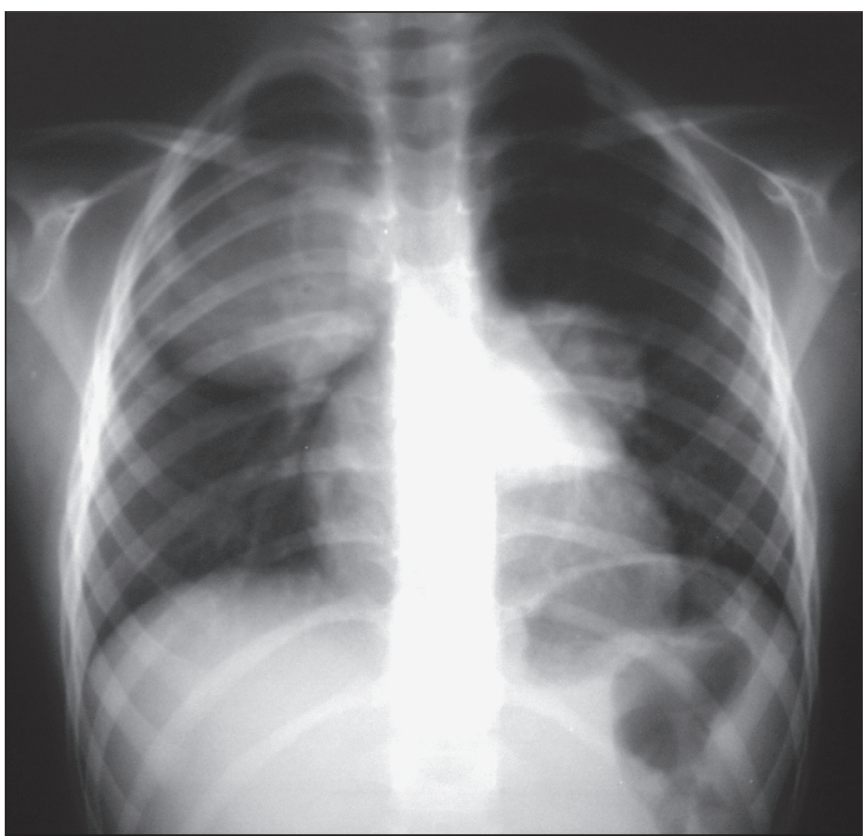

Figura 1 - Áreas de condensações nodulares focais com efeito de massa ("em bala de canhão") em terço superior do pulmão direito e médio esquerdo; seios costo-frênico livres; área cardíaca normal cefaléia nos últimos dois dias. Negava tosse e outros sintomas respiratórios. Ao exame, apresentava-se em regular estado geral, toxemiada, com palidez cutâneo-mucosa, perfusão periférica diminuída, respiração rítmica e regular, ausência de cianose, anictérica e febril $\left(39^{\circ} \mathrm{C}\right)$. A paciente mostrava adequação de peso e de estatura para a idade, freqüência cardíaca de 140 batimentos/minuto e frequiência respiratória de 32 incursões/minuto. A ausculta pulmonar evidenciava murmúrio vesicular diminuído difusamente e ausência de ruídos adventícios.

O raio-X do tórax de frente e perfil evidenciou áreas de condensações nodulares focais com efeito de massa em terço superior do pulmão direito e campo médio esquerdo (Figura 1). O hemograma apresentava 4.020 .000 hemácias; hemoglobina de 11,4g/dL; hematócrito 33\%; 14.400 leucócitos (1\% mielócitos, $2 \%$ metamielócitos, $9 \%$ bastonetes, $78 \%$ segmentados, $4 \%$ linfócitos típicos e $6 \%$ monócitos); os neutrófilos tinham granulações tóxicas finas. A proteína $\mathrm{C}$ reativa era de $279,8 \mathrm{mg} / \mathrm{dL}$ (normal até $5 \mathrm{mg} / \mathrm{dL}$ ).

Iniciou-se tratamento empírico para pneumonia com penicilina cristalina na dose de $200.000 \mathrm{U} / \mathrm{kg} / \mathrm{dia}$ endovenosa. A criança evoluiu com remissão da febre, melhora do estado geral já no terceiro dia e melhora radiológica no quarto dia de internação (Figura 2). Foi tratada por dez dias, com recuperação completa. A hemocultura não evidenciou crescimento de germes.

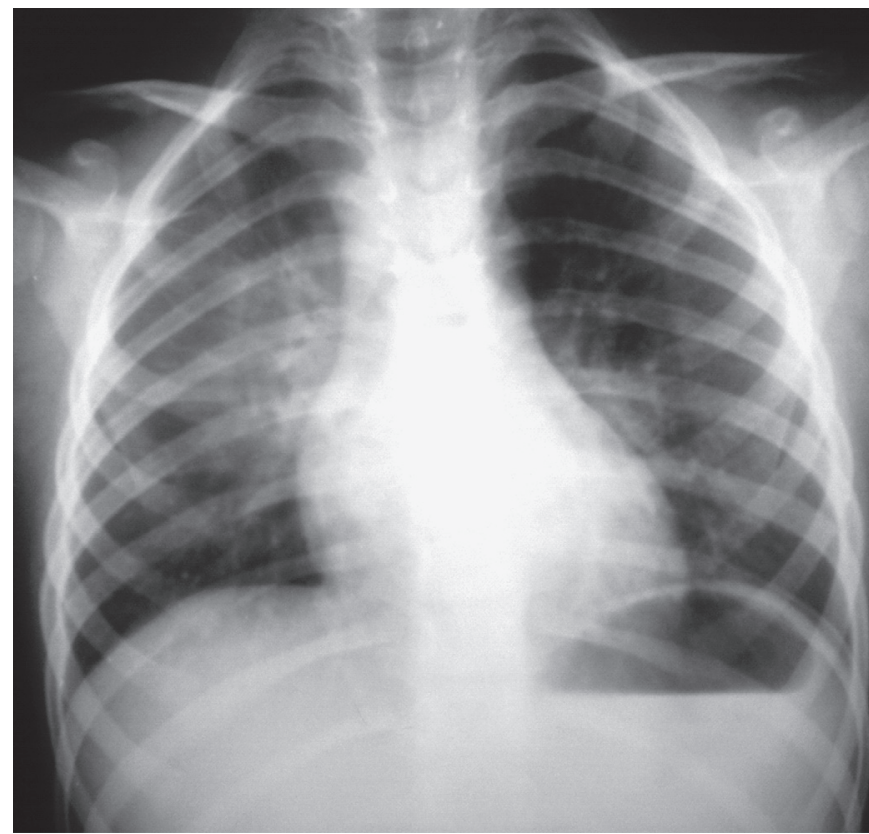

Figura 2 - Melhora nítida dos focos de condensação em lobo superior direito e justa-hilar esquerdo 


\section{Discussão}

Embora pouco freqüente, imagens radiológicas redondas ou ovais em crianças com sintomas infecciosos têm sido atribuídas a pneumonias. Parece que, inicialmente, há um processo exsudativo inflamatório alveolar, o qual se dissemina por extensão direta por meio dos poros de Kohn e dos canais de Lambert, conferindo distribuição não segmentar. Posteriormente, a propagação centrífuga do processo inflamatório peribrônquico determina o aparecimento de imagem segmentar ou lobar. O aspecto radiológico redondo constitui etapa precoce na evolução do processo. Nas crianças, os poros de Kohn são pobremente desenvolvidos e o tecido conectivo dos septos e alvéolos é escasso, o que contribui para produzir áreas confluentes mais compactas e de margens delimitadas, mais freqüentes nesta faixa etária ${ }^{(2,5-7)}$.

O principal agente etiológico das pneumonias é o Streptococcus pneumoniae ${ }^{(6,8,9)}$, embora outros, como Micoplasma pneumoniae, Clamidia pneumoniae, Legionela pneumophila, febre $\mathrm{Q}^{(1,2)}$, possam estar implicados em menor freqüência. No caso em questão, o tratamento foi dirigido empiricamente para o Streptococcus pneumoniae, tendo em vista que a pneumonia redonda tem sido, na literatura, relacionada a esta etiologia, embora a hemocultura não isolasse o agente.

Outro aspecto interessante deste caso foi a ausência de sinais propedêuticos de consolidação pulmonar e de repercussão respiratória. Em estudo que objetivou avaliar a importância da radiografia de tórax em crianças com febre sem sinais localizatórios e com leucocitose no hemograma, observou-se $19 \%$ de crianças com pneumonia sem propedêutica pulmonar ${ }^{(10)}$. Um aspecto interessante é a ausência de broncograma aéreo nestas imagens, dificultando o diagnóstico de pneumonia ${ }^{(7)}$.

\section{Referências bibliográficas}

1. Antón E. A frequent error in etiology of round pneumonia. Chest 2004;125:1592-3.

2. Zinkernagel AS, Schaffner A, Himmelman A. Photo Quiz. Round pneumonia due to Streptococcus pneumoniae. Clin Infect Dis 2001;32:1133-4.

3. Brasil. Ministério da Saúde. Assistência e controle das infecções respiratórias agudas. Brasília: Ministério da Saúde; 1994.

4. Ricetto AG, Zambom MP, Morcillo AM. Características de crianças com pneumonias atendidas no pronto-socorro. Rev Cienc Med Campinas 2003;12:55-62.

5. Souza EG, Nogueira MC, Amado D. Pneumonia redonda. J Pneumol 1990;16:178-80.

6. McLennan MK. Radiology rounds. Round pneumonia. Can Fam Physiciam 1998;44:757-9.

7. Wagner AL, Szabunio M, Hazlett KS, Wagner SG. Radiologic manifestations of round pneumonia in adults. Am J Roentgenol 1998;170:723-6.
Um diagnóstico diferencial importante para estas imagens radiológicas circulares é a neoplasia ${ }^{(9)}$. Lesões malignas, além das metástases, são extremamente raras em crianças; no entanto, dada sua relativa frequiência, a metástase de Tumor de Wilms deve ser considerada diante de quadros radiológicos similares ao aqui descrito ${ }^{(6)}$. Na paciente em questão, a hipótese de neoplasia não foi formulada inicialmente, pois o quadro infeccioso agudo foi acompanhado de leucocitose com desvio à esquerda, aumento da proteína $\mathrm{C}$ reativa e resposta eficaz clínica e radiológica à antibioticoterapia.

Também deve ser lembrado no diagnóstico diferencial, embora pouco freqüente na criança, o abscesso pulmonar resultante de processo supurativo e necrótico que se desenvolve em uma região circunscrita do parênquima pulmonar. A imagem radiológica pode ser a de velamento de conformação arredondada, que, por vezes, apresenta nível ar-líquido. As principais manifestações clínicas são tosse, prostração e febre, que pode chegar a $40^{\circ} \mathrm{C}$. O tratamento é essencialmente clínico, porém, a melhora do estado geral e da ausculta pulmonar ocorre em dez a 14 dias de tratamento e a resolução radiológica pode demorar oito semanas a seis meses. Naqueles casos em que a evolução após a antibioticoterapia não é satisfatória, cogita-se o tratamento cirúrgico. No caso relatado, pôde-se afastar esta hipótese diante da rápida melhora clínica e resolução radiológica ${ }^{(11-13)}$.

Pode-se concluir que, diante de imagem radiológica arredondada e associada a alterações laboratoriais compatíveis com quadro infeccioso, é razoável formular a hipótese de pneumonia redonda e aguardar a resposta clínica à antibioticoterapia, adiando procedimentos diagnósticos de maior complexidade e mais invasivos, mesmo porque o abscesso pulmonar e as neoplasias pulmonares são bem menos freqüentes do que as pneumonias nas crianças.

8. Camargos PA, Ferreira CS. On round pneumonia in children. Pediatr Pulmonol 1995;20:194-5.

9. Jardim C, Ferreira JC, Takagaki TY, Souza R. Pneumonia redonda/pneumonia pseudotumoral. Rev Assoc Med Bras 2003;49:240.

10. Bachur R, Perry H, Harper MB. Occult pneumonias: empiric chest radiographs in febrile children with leukocytoses. Ann Emerg Med 1999;33: 166-73.

11. Nakaie CMA. Abscesso pulmonar. In: Marcondes E, Vaz FAC, Ramos JCA, Okay Y, editores. Pediatria básica. 9a ed. São Paulo: Sarvier; 2004. p. 187-91.

12. Nakaie CMA. Abscesso pulmonar. In: Rozov T, editor. Afecções respiratórias não específicas em pediatria. São Paulo: Sarvier, $1^{\text {a }}$ ed. 1986. p. 115-27.

13. Nakaie CMA, Cardieri JMA, Rozov T. Abscesso pulmonar: relato de 26 casos. Pediatria (São Paulo) 1985;7:132-6. 